\title{
CULTURA DE SEGURIDAD DEL PACIENTE DE LAS ENFERMERAS ASISTENCIALES DEL HOSPITAL REGIONAL DE ICA - 2017
}

\author{
PATIENT SAFETY CULTURE OF THE ASSISTANT NURSES OF THE ICA REGIONAL HOSPITAL - 2017"
}

\author{
Norma Pastor Ramírez ${ }^{1, a, c}$, Myriam Arias Patiño ${ }^{2, a, d}$ Walter Alberto Azula Aguinaga ${ }^{3, b}$ \\ Norma Pastor Ramírez ${ }^{1, a, c}$, Myriam Arias Patiño ${ }^{2, a, d}$ Walter Alberto Azula Aguinaga \\ ${ }^{1}$ Facultad de Enfermería. Universidad Nacional San Luis Gonzaga. Ica, Perú \\ ${ }^{2}$ Hospital Regional de Ica. Ica, Perú \\ ${ }^{3}$ Facultad de Medicina Humana, Universidad Nacional San Luis Gonzaga. \\ ${ }^{a}$ Lic. en Enfermería. ${ }^{b}$ Lic. en Ciencias Biológicas ${ }^{c}$.Magister en Salud Pública ${ }^{d}$ Especialista en Unidad de Cuidados \\ Intensivos Neonatales
}

\section{RESUMEN}

Objetivo: Determinar las competencias en cultura de seguridad del paciente de las enfermeras asistenciales del hospital Regional de Ica. Material y Métodos: El diseño del estudio es Observacional, Descriptivo, Aplicada y cuantitativa. Muestra constituida por 102 enfermeras. Utilizando un cuestionario para enfermeras hospitalarias sobre seguridad de los pacientes (CEHSP) Resultados: Obtenidos en relación a las dimensiones estudiadas fueron: en aprendizaje organizacional / mejora continua $52,0 \%$, trabajo en equipo $52,0 \%$, apoyo de la gerencia del hospital $38,2 \%$, Eventos notificados $44,1 \%$, Percepción de seguridad $12,7 \%$, Dotación de personal 7,8\%, Expectativas y acciones que favorecen a la seguridad 20,6\%, Feedback y comunicación sobre errores $47,1 \%$, Franqueza en la comunicación $21,6 \%$, Respuesta no punitiva a los errores $12,7 \%$, Problemas en cambio de turno y transiciones 13,7\%, Trabajo en equipo entre unidades 5,9\%. Conclusiones: El estudio realizado según se refleja en las dimensiones en cuyas respuestas se aprecia que requieren un plan de mejora ya que no superan el $75 \%$ de las respuestas de acuerdo a la valoración, por lo tanto ninguno de los ítems puede ser considerado una fortaleza. Las debilidades más destacadas son trabajo en equipo entre unidades, seguida de la falta de dotación de personal, la respuesta no punitiva ante los errores acompañados de la dimensión percepción de seguridad, la cual evidencia la cultura de seguridad hospitalaria en nuestra institución.

Palabras clave: Cultura de seguridad del paciente, enfermeras.

\section{SUMMARY}

Objective: To determine the competencies in the patient safety culture of the nurses of the Regional Hospital of Ica.Methods:Study design Observational, Descriptive, Applied and quantitative and Results: in relation to the dimensions were obtained in organizational learning/continuous improvement $52.0 \%$, teamwork $52.0 \%$, support from hospital management $38.2 \%$, Notified events $44.1 \%$, Perception of safety $12.7 \%$, Staffing $7.8 \%$, Expectations and actions that favor safety $20.6 \%$, Feedback and communication about errors $47.1 \%$, penness in communication $21.6 \%$, Nonpunitive response to errors $12.7 \%$, Problems in turn shift and transitions $13.7 \%$, Teamwork between units $5.9 \%$. Conclusions: The study carried out in our institution as reflected in the dimensions in whose responses we can see that they require an improvement plan since they do not exceed $75 \%$ of the answers according to the assessment, therefore none of the items can be considered strength. The most outstanding weaknesses are teamwork between units, followed by the lack of staffing, the non-punitive response to errors accompanied by the perception of safety dimension, which evidences the culture of hospital safety in our institution.

Keywords: Patient safety culture, nurses 


\section{INTRODUCCIÓN}

Hay pocos estudios conocidos que hagan referencia exclusivamente a servicios de hospitalización. De este modo el presente estudio mide el grado de cultura de seguridad del paciente de las enfermeras asistenciales del Hospital Regional de Ica con lo cual se pretende incrementar el bagaje científico local y otorgar la suficiente información que permita fortalecer las dimensiones destinadas a mejorar la calidad del servicio que se otorga al paciente.

Debemos citar la frase "primun non nocere" de Hipócrates (460-377 a.C.) como la norma que regula el ejercicio en el ámbito médico, sin embargo, no fue hasta que Florence Nightingale (1854) quien calculando la tasa de mortalidad en los hospitales militares de esa época (guerra de Crimea) llegan a la conclusión que la mejora en los métodos sanitarios empleados, produce una disminución del número de muertes. La seguridad del paciente es el principio fundamental de la atención sanitaria y un componente crítico de la gestión de la calidad. Se sabe que las intervenciones en el proceso de atención de la salud deteriorada se realizan para beneficiar a los pacientes, sin embargo, un importante porcentaje de éstas, pueden causar daño, justamente por la complejidad en que se desarrollan estas acciones. La seguridad del paciente, dimensión esencial de la calidad asistencial y un derecho de los pacientes reconocido por organismos como la Organización Mundial de la Salud (OMS), la Organización Panamericana de la Salud (OPS) y el Comité de Sanidad del Consejo de Europa que junto con diversas agencias $y$ organismos internacionales $(1,2)$, recomiendan a los diferentes gobiernos situar la seguridad del paciente en el centro de todas sus políticas sanitarias, invitándoles a proponer planes y actividades de mejora así como aprobar medidas legislativas para que en cada institución con la participación de los profesionales se puedan reducir los eventos adversos evitables.

La Cultura de seguridad suele definirse como "el producto de los valores, actitudes, competencias y patrones de comportamiento individuales y colectivos que determinan el compromiso, el estilo y la capacidad de una organización de salud o de un sistema de seguridad" (3) , [....Se entiende que dicha cultura es un constructo compuesto por dimensiones o subculturas, tales como el trabajo en equipo, comunicación, aprendizaje, etc. La medición de la cultura de seguridad tiene como objetivo cuantificar las debilidades y fortalezas en cada subcultura, para favorecer las intervenciones de mejora, tanto de las percepciones y actitudes, como de los incidentes de seguridad. Pese a que hay pocos estudios concluyentes sobre la relación entre cultura de seguridad deficiente, y la alta incidencia de errores o eventos adversos, se sabe que este es un factor que contribuye a su ocurrencia...]. (4). Construir una cultura de seguridad (CS) en un centro hospitalario es la clave fundamental para conseguir que la seguridad del paciente, forme parte de la metodología de trabajo, de mejora de la calidad en los servicios asistenciales, para ello es necesario conocer cuál es la situación de partida a fin de saber cuáles son los aspectos que conviene priorizar. 


\section{MATERIAL Y METODOS:}

La población de estudio fueron las enfermeras asistenciales que laboran en el Hospital Regional de Ica-MINSA, Departamento de Ica, Perú. El número de enfermeras que trabajan en el área asistencial son en promedio 210 de las cuales 102 fue la 'población incluida según el tamaño muestral resultante con un margen de error del $7 \%$ y un nivel de confianza del 95\%.

El estudio es de tipo Observacional, Descriptivo, transversal, Aplicada y cuantitativa. Se utilizó el cuestionario CEHSP (5) (Cuestionario para enfermeras hospitalarias sobre la Seguridad de los Pacientes), este último fue a su vez adaptado y validado al contexto español por Ammouri AA, Tailakh A., Muliira JK, Geethakrishnan R, Al Kindi SN. Patient safety culture among nurses. partiendo del cuestionario original titulado "Hospital

\section{RESULTADOS}

Se presentan los resultados divididos en dimensiones clasificados de acuerdo al cuestionario aplicado. En cada sección se
Survey on Patient Safety" y que fue desarrollado por la Agency for Health care Research and Quality (AHRQ) para la recogida de datos. Se trata de una herramienta fiable y válida para la medición de los valores, actitudes, percepciones, competencias, comportamientos y condiciones laborales de las enfermeras asistenciales relacionadas con la seguridad del paciente.

El cuestionario sobre seguridad de los pacientes el cual está adaptado y dirigido a profesionales enfermeros asistenciales, es autoadministrable, consta de 4 páginas con un total de 61 preguntas, su acceso es libre y se encuentra disponible en la página web. El cuestionario cuenta con 12 dimensiones relacionadas con la percepción de los profesionales sobre la cultura de la Seguridad del Paciente

Tabla N01 Variable de datos Sociodemográficos

\begin{tabular}{lcc} 
Variable Sociodemográficos & $\mathbf{N}^{\circ}$ & $\%$ \\
\hline EDAD & & 9,80 \\
\hline $25-30$ & 10 & 33,33 \\
\hline $31-40$ & 34 & 27,46 \\
\hline $41-50$ & 28 & 29,41 \\
\hline 51 A Más & 30 & \\
\hline SEXO & & 82,40 \\
\hline Femenino & 84 & 17,60
\end{tabular}


Tabla $\mathrm{N}^{\circ} 02$ Variable de la actividad profesional

\begin{tabular}{lcc} 
Experiencia Profesional & $\mathbf{N}^{\circ}$ & $\%$ \\
\hline 0-5 Años & 17 & 16,70 \\
\hline 6-10 Años & 23 & 22,50 \\
\hline 11 A 15 Años & 14 & 13,70 \\
\hline 16-20 Años & 21 & 20,60 \\
\hline 21-30 Años & 19 & 18,70 \\
\hline 31 a Mas & 8 & 7,80 \\
\hline Servicio en la que Trabaja & $\mathbf{N}^{\circ}$ & $\%$ \\
\hline Cirugía & 17 & 16,70 \\
\hline Medicina & 19 & 18,60 \\
\hline Centro Quirúrgico & 4 & 3,90 \\
\hline Emergencia & 20 & 19,60 \\
\hline Neonatología & 7 & 6,90 \\
\hline Pediatría & 4 & 3,90 \\
\hline Unidad de Cuidaos Intensivos Neonatales & 15 & 14,70 \\
\hline Unidad de Cuidaos Intensivos Pediátricos & 6 & 5,90 \\
\hline Unidad de Cuidados Intensivos Adultos & 10 & 9,80
\end{tabular}

Tabla Nº3 Variable de la Actividad Profesional

\begin{tabular}{lcc} 
Condiciones Laborales & $\mathbf{N}^{\circ}$ & $\%$ \\
\hline Nombrado & 75 & 73,50 \\
\hline Contratado & 27 & 26,50 \\
\hline Turnos de Trabajo & $\mathbf{N}^{\circ}$ & $\%$ \\
\hline Turno Mañana & 35 & 34,30 \\
\hline Rotativos & 67 & 65,70 \\
\hline Cursos de Inducción & $\mathbf{N}^{\circ}$ & $\%$ \\
\hline Si recibió & 16 & 15,70 \\
\hline No recibió & 86 & 84.30 \\
\hline Acompañamiento en el Servicio & $\mathbf{N}^{\circ}$ & $\%$ \\
\hline Si & 18 & 17,60 \\
\hline No & 84 & 84,90 \\
\hline Titulo Profesional más Alto & $\mathbf{N}^{\circ}$ & $\%$ \\
\hline Segunda especialidad & 66 & 64,70 \\
\hline Magister & 34 & 33,30 \\
\hline Doctor & 2 & 2,00 \\
\hline
\end{tabular}


Tabla № 04 Categorías de recodificación para calcular las frecuencias

\begin{tabular}{|c|c|c|c|c|c|}
\hline $\begin{array}{c}\text { Nuevas } \\
\text { Categorías } \\
\text { (Recodificadas) }\end{array}$ & \multicolumn{2}{|c|}{ Negativo } & Neutral & \multicolumn{2}{|c|}{ Positivo } \\
\hline \multirow{2}{*}{$\begin{array}{c}\text { Categorías } \\
\text { Originales en } \\
\text { ítems }\end{array}$} & $\begin{array}{c}\text { Muy en } \\
\text { desacuerdo }\end{array}$ & Desacuerdo & Indiferente & $\begin{array}{c}\text { De } \\
\text { acuerdo }\end{array}$ & $\begin{array}{l}\text { Muy de } \\
\text { Acuerdo }\end{array}$ \\
\hline & Nunca & Casi nunca & A veces & $\begin{array}{c}\text { Casi } \\
\text { Siempre }\end{array}$ & Siempre \\
\hline
\end{tabular}

Tabla № 5 Consolidado según dimensiones Fortalezas y debilidades

Requieren un plan de mejora ya que no superan el $75 \%$ en las respuestas de acuerdo a la valoración,

\begin{tabular}{|c|c|c|c|c|c|}
\hline \multirow{3}{*}{ CONSOLIDADO } & \multirow{2}{*}{\multicolumn{2}{|c|}{ FORTALEZA }} & \multicolumn{2}{|c|}{ PARA } & \multirow{3}{*}{ TOTAL } \\
\hline & & & \multicolumn{2}{|c|}{ MEJORAR } & \\
\hline & $\mathbf{N}$ & $\%$ & $\mathbf{N}$ & $\%$ & \\
\hline Aprendizaje organizacional / Mejora continua & 53 & 52,0 & 49 & 48,0 & 102 \\
\hline Trabajo en equipo & 53 & 52,0 & 49 & 48,0 & 102 \\
\hline
\end{tabular}

Tabla $N^{\circ} 6$ Debilidades u oportunidades de mejora ( $>50 \%$ de las respuestas de carácter negativo)

\begin{tabular}{lccccc}
\multirow{2}{*}{ CONSOLIDADO SEGÚN DIMENSIONES } & \multicolumn{2}{c}{ FORTALEZA } & \multicolumn{2}{c}{ PARA } \\
& \multicolumn{2}{c}{ MEJORAR } & \multirow{2}{*}{ TOTAL } \\
\cline { 2 - 5 } & $\mathbf{N}$ & $\%$ & $\mathbf{N}$ & $\%$ & \\
\hline Apoyo de la gerencia del hospital & 39 & $\mathbf{3 8 , 2}$ & 63 & $\mathbf{6 1 , 8}$ & 102 \\
\hline Eventos notificados & 45 & $\mathbf{4 4 , \mathbf { 1 }}$ & 57 & $\mathbf{5 5 , 9}$ & 102 \\
\hline Percepción de seguridad & 13 & $\mathbf{1 2 , 7}$ & 89 & $\mathbf{8 7 , 3}$ & 102 \\
\hline Dotación de personal & 8 & $\mathbf{7 , 8}$ & 94 & $\mathbf{9 2 , 2}$ & 102 \\
\hline Expectativas y acciones que favorecen a la & 21 & $\mathbf{2 0 , 6}$ & 81 & $\mathbf{7 9 , 4}$ & 102 \\
\hline Feedback y comunicación sobre errores & 48 & $\mathbf{4 7 , 1}$ & 54 & $\mathbf{5 2 , 9}$ & 102 \\
\hline Franqueza en la comunicación & 22 & $\mathbf{2 1 , 6}$ & 80 & $\mathbf{7 8 . 4}$ & 102 \\
\hline Respuesta no punitiva a los errores & 13 & $\mathbf{1 2 , 7}$ & 89 & $\mathbf{8 7 , 3}$ & 102 \\
\hline Problemas en cambio de turno y & 14 & $\mathbf{1 3 , 7}$ & 88 & $\mathbf{8 6 , 3}$ & 102 \\
\hline Transiciones & & & & & \\
\hline Trabajo en equipo entre unidades & 6 & $\mathbf{5 , 9}$ & 96 & $\mathbf{9 4 , 1}$ & 102 \\
\hline
\end{tabular}




\section{Tabla $\mathbf{N}^{\circ} 7$}

Los indicadores para cada dimensión se han calculado aplicando la media de las respuestas obtenidas para cada categoría.

Para identificar una pregunta o una dimensión como una fortaleza del personal profesional de enfermería del hospital Regional de Ica (HRI) se han considerado los siguientes criterios:

$\mathbf{2 7 5 \%}$ de respuestas positivas ("de acuerdo/muy de acuerdo" o "casi siempre/siempre") a preguntas formuladas en positivo.

$\mathbf{2 7 5 \%}$ de respuestas negativas ("en desacuerdo/muy en desacuerdo" o "nunca/raramente") a preguntas formuladas en negativo.

Por el contrario, para clasificar una pregunta o una dimensión como debilidad u oportunidad de mejora se han empleado los criterios que se muestran a continuación:

$\geq 25 \%$ de respuestas negativas ("en desacuerdo/muy en desacuerdo" o "nunca /raramente") a preguntas formuladas en positivo.

$\geq 50 \%$ de respuestas positivas ("de acuerdo/muy de acuerdo" o "casi siempre/siempre") a preguntas formuladas en negativo.

Tabla 7 Cuadro Resumen de Fortaleza y Debilidades u oportunidades

\begin{tabular}{cllllll} 
ENCUESTADO & ITEM A & ITEM B & ITEM C & ITEM D & $\begin{array}{l}\text { PORCENTAJE } \\
(+)\end{array}$ & INDICADOR \\
\hline $\mathbf{1}$ & Positivo & Positivo & Positivo & Neutral & $\mathbf{7 5 \%}$ & Fortaleza \\
\hline $\mathbf{2}$ & Neutral & Negativo & Positivo & Neutral & $\mathbf{2 5 \%}$ & Mejora \\
\hline 3 & Positivo & Positivo & Neutral & Negativo & $\mathbf{5 0 \%}$ & Mejora \\
\hline 4 & Positivo & Positivo & Neutral & Positivo & $\mathbf{7 5 \%}$ & Fortaleza \\
\hline 5 & Neutral & Negativo & Neutral & Negativo & $\mathbf{0 \%}$ & Mejora \\
\hline
\end{tabular}

\section{DISCUSIÓN DE LOS RESULTADOS: En} los últimos años, los estudios sobre cultura de seguridad en el ámbito internacional, sobre todo en Latinoamérica, se han incrementado sustancialmente. En este trabajo el instrumento utilizado permitió contar con una primera aproximación diagnóstica a la situación del hospital evaluado. Datos Sociodemográficos, ( tabla $N^{\circ} 1$ ) el promedio de edad es 44,3 años en un rango de 25 a 51 y más, siendo el de mayor predominancia el sexo femenino, se incluyó la participación de las Enfermeras que trabajan en diferentes unidades de la institución en mención. En nuestro estudio, la media del tiempo ejerciendo como enfermera fue de 16, 5 años, con una oscilación entre 1 año y 40 años, la Condición Laboral del personal de enfermería nombrado con un total de
$73,5 \%$, desempeñándose en los turnos de trabajo rotativos en su mayoría con un total de $65,7 \%$, de las cuales al momento de ser rotados o programados en los servicios diferentes no recibieron inducción con un promedio de $84,3 \%$, no recibió acompañamiento en el servicio con un promedio $84,9 \%$; lo que es un reflejo de movilidad interna a la que están acostumbradas las enfermeras en los hospitales públicos ( tabla $\mathrm{N}^{\circ} 2$ y 3 ). Las respuestas del cuestionario se recodificaron en tres categorías (tabla $\mathrm{N}^{\circ} 4$ ) para calcular las frecuencias de cada categoría para todas las preguntas y dimensiones. La comunicación dentro del equipo de salud es un pilar importante dentro del desarrollo y ejecución de medidas que propendan por una atención segura, es así como la confianza dentro 
del personal y el seguimiento de los conductos regulares para la notificación de eventos que ponen en riesgo al paciente, cumple un papel importante para la formación de una verdadera cultura de seguridad del paciente. Hay que tener en cuenta que un personal que confía en sus conocimientos y es capaz de reconocer sus errores, siempre desarrollará líneas de comunicación claras que asegurarán un manejo adecuado de los incidentes ocurridos dentro del servicio y dará lugar a verdaderas oportunidades de mejora y a la formulación de planes de acción efectivos y coherentes con la situación real de la unidad. En general, nuestros resultados se asemejan más a los obtenidos por Martínez V. (6). "Problemas en las transferencias de pacientes o cambios de turno" obtuvieron el $57,8 \%$ cada uno respectivamente como también nuestros resultados no se asemejan a ninguna de las referencias revisadas. con el objetivo de conocer detalladamente la asociación entre estos factores y la cultura de seguridad, no solo se ha realizado una inferencia estadística con las respuestas positivas como en el estudio al que hacemos mención, sino que también se ha realizado con las respuestas negativas. Igualmente, siguiendo la propuesta de la $A H R Q$, se han recogido las fortalezas y las

\section{CONCLUSIONES:}

1. La caracterización de la cultura de seguridad de los pacientes referidos en el Hospital Regional de Ica (HRI) permitió evidenciar la necesidad de implementar actividades de mejora para favorecer las prácticas seguras y el trabajo en equipo.

2. Los resultados del estudio también nos muestra muy claramente que cuando se debilidades percibidas por las enfermeras del HRI con el objetivo de que puedan ser consideradas para potenciar y mejorar la cultura de seguridad en el mismo. En resumen, según la investigación las fortalezas y debilidades (tabla $N^{\circ} 5$ ), en el clima de seguridad del paciente percibidas por las enfermeras del Hospital Regional de Ica, refleja en las dimensiones cuyas respuestas se aprecia que requieren un plan de mejora ya que no superan el $75 \%$ en las respuestas de acuerdo a la valoración, por lo tanto ninguno de los ítems puede ser considerado una fortaleza.

Las debilidades (tabla $N^{\circ} 6$ ) más destacadas son trabajo en equipo entre unidades, seguida de la falta de dotación de personal, la respuesta no punitiva ante los errores acompañados de la dimensión percepción de seguridad, la cual evidencia la cultura de seguridad hospitalaria en nuestra institución.

Los indicadores para cada dimensión se han calculado aplicando la media de las respuestas obtenidas para cada categoría. Para identificar una pregunta 0 una dimensión como una fortaleza del HRI se han considerado los criterios descritos en la tabla $\mathrm{N}^{\circ} 7$.

incrementa la exigencia laboral, los intereses institucionales superan a los de la seguridad del paciente la cual denota que se trabaja en condiciones desfavorables sin importar el resultado ni los objetivos de su trabajo. Otros factores como remplazos de personal, quizá sin las competencias esperadas son considerados como factores que

Rev. enferm. vanguard. 2019; 7(2) 
impiden que se pueda brindar una mejor atención al paciente.

3. En cuanto a dimensiones de la cultura de seguridad a nivel de Hospital, se detectó la falta de una comunicación oportuna dentro de los miembros del equipo de salud dentro de un mismo servicio y unidad, ya que la comunicación oportuna y la retroalimentación en los errores en la atención permiten entablar alternativas de solución y prevenir futuros eventos adversos en el cuidado del paciente.

\section{Correspondencia:}

Mg. Norma Pastor Ramírez.

Correo electrónico: npr047@ gmail.com

Celular: 969528647

\section{REFERENCIA BIBLIOGRAFICA}

1. Madrid, Ministerio de Sanidad y Consumo Seguridad del paciente. Estudio Nacional sobre los efectos adversos ligados a la Hospitalización. ENEAS 2005. Informe. Madrid; 2006.

2. España, Ministerio de Sanidad y Política Social. Estudio IBEAS. Prevalencia de efectos adversos en hospitales de Latinoamérica. ; 2010.

3. Health and Safety. Commission Advisory Committee on the Safety of Nuclear Installations. Organizing for safety: third report of the ACSNI study
4. El análisis descriptivo de los datos permitió identificar la percepción de los profesionales de enfermería acerca de la cultura de seguridad presente en nuestra institución sugieren una comprensión más crítica de los directivos teniendo en cuenta que los constructos relacionados con estos temas obtuvieron resultados negativos la cual representa una amenaza para la seguridad del paciente.

group on human factors. Sudbury: HSE Books; 1993.

4. Sammer CE, Lykens K, Singh KP, Mains DA, Lackan NA. What is patient safety culture? A review of the literature. J Nurs Scholarsh; 2010.

5. Madrid, Ministerio de Sanidad y Política Social. Análisis de la cultura sobre seguridad del paciente en el ámbito hospitalario del Sistema Nacional de Salud Español. Madrid; 2009

6. Martínez V. Cultura de seguridad del paciente, en enfermeras del Servicio de Centro Quirúrgico y Recuperación del Hospital Nacional Dos de Mayo. Lima, Perú; 2015

Recibido: $13 / 08 / 19$

Aprobado para publicación: 20/11/19 